\title{
Regulação de acesso da urgência e emergência oftalmológica em um hospital universitário
}

\section{Regulation of access in the urgency and emergency ophthalmological in university hospital}

\author{
Christine Lobato Bemerguy ${ }^{1}$ \\ Orcid: https://orcid.org/0000-0003-4170-6440
}

Isabelle Christine Vieira da Silva Martins ${ }^{2}$

Orcid: https://orcid.org/0000-0002-6718-9202

\author{
João Farias Guerreiro ${ }^{3}$ \\ Orcid: https://orcid.org/0000-0003-1979-3656
}

\begin{abstract}
Resumo
Introdução: A regulação de acesso nos serviços oftalmológicos, quando estruturada com processos, protocolos e fluxos bem estabelecidos garante a organização do acesso, da oferta e da demanda dos serviços, o que oferece qualidade e resolutividade na assistência em tempo hábil à população. Objetivos: Identificar a Regulação do acesso à assistência das Urgências e Emergências (U/E) oftalmológicas segundo os profissionais do setor em um Hospital Universitário em Belém, PA. Materiais e métodos: Utilizou-se um questionário com quinze perguntas abertas e fechadas sobre conhecimento da regulação de acesso para os profissionais do setor de oftalmologia. A análise quantitativa foi realizada com base na estatística descritiva. Resultados: Foram avaliados 29 profissionais, sendo em sua maioria com tempo de atuação de 1 a 5 anos na Oftalmologia (65,5\%), no Hospital $(72,4 \%)$ e no setor de Oftalmologia do Hospital (75\%). Dos entrevistados, 58,6\% acreditam que exista um espaço adequado para o atendimento do paciente em situação de U/E, 61,5\% disseram desconhecer protocolo assistencial para o atendimento do paciente em situação de U/E. Quanto à priorização do atendimento de U/E, 82,7\% afirmaram acontecer. E 71,4\% afirmaram que o serviço de Oftalmologia tem acesso imediato ao Centro Cirúrgico. 68,9\% acreditam que têm capacidade de atender U/E nas 24 horas, 82,7\% desconhecem o número de U/E em Oftalmologia atendidos e $51,7 \%$ têm conhecimento do fluxo externo de demanda do atendimento. Conclusões: A maioria dos profissionais reconhecem a importância das U/E Oftalmológicas para comunidade, desconhecem os protocolos, porém destacam a necessidade de melhorias nos processos de trabalho.
\end{abstract}

Palavras-chave: acesso universal aos serviços de saúde; sistema único de saúde; política de saúde

\begin{abstract}
Background: The regulation of access in ophthalmic services, when structured with wellestablished processes, protocols and flows, guarantees the organization of access, supply and demand of services, offering quality and resoluteness in care in a timely manner to the population. Objectives: To identify the regulation of access to care in Ophthalmologic Emergency and Emergency (U/E) Services according to sector professionals at University Hospital in Belém, PA. Materials and methods: We used questionnaire with fifteen open and closed questions about knowledge of access regulation for professionals in the ophthalmology sector. A quantitative analysis was performed based on descriptive statistics. Results: Twentynine professionals were evaluated, most of them with experience of 1 to 5 years in Ophthalmology $(65.5 \%)$, in the Hospital $(72.4 \%)$ and in the Ophthalmology sector of the
\end{abstract}

\footnotetext{
${ }^{1}$ Universidade Federal do Pará, Programa de Pós-graduação em Neurociências e Biologia Celular, Brasil. E-mail: christinelobato@yahoo.com.br

${ }^{2}$ Universidade Federal do Pará, Programa de Pós-graduação em Neurociências e Biologia Celular, Brasil. E-mail: isabellecvsm@gmail.com

${ }^{3}$ Universidade Federal do Pará, Programa de Pós-graduação em Neurociências e Biologia Celular, Brasil. E-mail: joaofg@ufpa.br
} 
Hospital (75\%). Of the interviewees, $58.6 \%$ believe that an adequate space for patient care in $\mathrm{U} / \mathrm{E}$ situation, $61.5 \%$ said were unaware of the care protocol for patient care in U/E situation. As for the prioritization of U/E care, $82.7 \%$ claimed to happen. In addition, $71.4 \%$ stated that the Ophthalmology service has immediate access to the Surgical Center. $68.9 \%$ believe capacity to serve U/E within 24 hours, $82.7 \%$ not know the number of U/E in Ophthalmology attended and $51.7 \%$ are aware of the external flow of demand for the service. Conclusions: The majority professionals recognize the importance of Ophthalmologic U/E for the community, are unaware of the protocols, but highlight the need for improvements in work processes

Keywords: universal access to health care services; unified health system; health policy word.

\section{Introdução}

O perfil de morbimortalidade da população tem apresentado aumento de doenças crônicas e causas externas. Refletindo nitidamente nos serviços de saúde, sobretudo nos de urgência e emergências, pois aumenta o número de atendimentos, os gastos com assistência e a reabilitação ${ }^{1}$. A OMS (2000) estima que ocorrem cerca de 55 milhões de acidentes oculares por ano, sendo que destes 200.000 provocam lesões abertas do globo ocular. A perda visual causa um impacto à sociedade em diversos níveis e os custos econômicos da cegueira é enorme tanto para o poder público quanto para a coletividade ${ }^{2}$.

Assim como se alteram os padrões epidemiológicos, a oferta dos serviços de saúde também deve sofrer adequações em igual proporção, a fim de que sejam atendidas as necessidades emergentes; e o Sistema Único de Saúde (SUS) insere-se como um mecanismo de preservação da sociedade $^{3}$.

A Regulação das Ações de Saúde, a partir de acordos prévios entre gestores municipais, instituições de saúde vinculadas ao SUS e representantes da comunidade, visa facilitar o acesso adequado de forma responsável e ágil aos recursos assistenciais necessários para cada demanda, com critérios regulatórios pactuados e transparentes ${ }^{4}$.

No entanto, são constantes os relatos de longas filas de espera, falta de profissionais capacitados, estruturas sucateadas dos hospitais, e pessoas sofrendo sem esses atendimentos, o que revela a deficiência do sistema de urgência oftalmológica no Brasil e a necessidade de estimular a melhoria desse serviço ${ }^{5}$.

No contexto do SUS, o atendimento aos casos de urgência e emergência encontra-se dentro do processo de regulação dos serviços de saúde. É uma experiência que articula duas novas práticas: o uso da Tecnologia da Informação na gestão do SUS e no atendimento ao cidadão, e a implantação do processo regulatório ${ }^{6}$.

Atualmente, $\quad \mathrm{o}$ adequado atendimento oftalmológico vem sendo impactado negativamente com (I) deficiência dos serviços de saúde destinados às urgências e emergências oftalmológicas, (II) ao processo de regulação dos mesmos e ao (III) desconhecimento dos profissionais de saúde. Nesse contexto, este estudo pretende analisar a regulação do acesso à assistência da urgência e emergência em oftalmologia em um Hospital Universitário segundo os profissionais do setor.

\section{Materiais e Métodos}

\section{Amostra e tipo de estudo}

Trata-se de um estudo observacional, descritivo e abordagem quantitativa realizado no setor de Oftalmologia em um Hospital Universitário em Belém, PA. A população do estudo foi composta pelos 29 profissionais que atuam no setor de Oftalmologia do Hospital, sendo 3 enfermeiros, 14 médicos, 6 técnicos de enfermagem, 2 auxiliares de enfermagem e 4 técnicos administrativos.

Este estudo foi aprovado pelo Comitê de Ética da Universidade Federal do 
Pará com CAAE: 37752920.3.0000.0017. Antecedendo à coleta dos dados ocorreu a assinatura do Termo de Consentimento Livre e Esclarecido.

\section{Delineamento da pesquisa}

Para a coleta de dados foi utilizado o Questionário de Avaliação da Regulação das Urgências e Emergências Oftalmológicas. O instrumento de pesquisa elaborado pelos pesquisadores é um questionário semiaberto com 15 perguntas abertas e fechadas, aplicado entre janeiro e maio de 2021. No questionário foram abordadas as questões como identificação dos participantes, conhecimento dos profissionais sobre Urgência/Emergência Oftalmológica e capacidade instalada do setor e fatores determinantes para realização do atendimento em Urgência/Emergência em oftalmologia nas 24 horas.

Em relação à capacidade do Hospital atender U/E nas 24 horas visa identificar se com a estrutura atual o hospital consegue atender essas urgências no tempo esperado. Sobre a importância do Hospital atender U/E nas 24 horas, objetiva compreender se os profissionais consideram a necessidade da população de ser atendida no menor tempo possível, o que leva à diminuição do prognóstico de cegueira previnível. No que se refere aos equipamentos existentes no $\mathrm{HU}$, visa avaliar se durante a assistência os profissionais conseguem realizar os exames necessários ao usuário com esses instrumentos.

O conhecimento sobre o número de atendidos na U/E por parte dos profissionais é um importante mecanismo de mensuração da necessidade de manter o serviço funcionante. Além disso, o conhecimento do fluxo externo de demanda no atendimento visa avaliar se o profissional conhece o percurso do paciente até o hospital, o tempo de espera para o atendimento, bem como a necessidade de melhorias na rede de atenção em oftalmologia.
Ao questionar sobre a existência de um espaço adequado para atendimento objetiva-se compreender a visão dos profissionais em relação ao espaço destinado para esse atendimento, bem como, a necessidade de melhorias. Sobre avaliar o conhecimento da existência de protocolos assistenciais, sabe-se a importância na qualidade do serviço prestado, visto que esses direcionam o profissional para realizar um tratamento seguro e padronizado ao usuário. Quanto à prioridade no atendimento e à existência de acesso imediato ao centro cirúrgico, pretende-se identificar se o profissional compreende a necessidade de atender imediatamente o usuário e se isso acontece no hospital.

Sobre as mudanças a serem realizadas segundo os profissionais do setor, para melhorias no serviço, buscou-se descobrir quais as mudanças eles compreendiam ser necessárias para adequar o hospital ao atendimento de excelência ao usuário.

Os participantes da pesquisa foram abordados, em um local reservado, sobre a pesquisa e então para aqueles que aceitaram participar foi apresentado o questionário impresso para ser autorrespondido. Os sujeitos da pesquisa tiveram sua dignidade e autonomia preservadas e poderiam desistir de participar a qualquer momento, sendo garantido a sua liberdade de contribuir para o estudo, através de manifestação expressa, livre e esclarecida.

\section{Critérios de Inclusão e Exclusão}

Foram considerados os seguintes critérios de inclusão: profissionais que atuam na assistência oftalmológica do hospital; sem distinção de vínculo empregatício, mas que estejam na atuação e maiores de 18 anos. Para os critérios de exclusão, profissionais que não atuam na assistência oftalmológica no hospital e estar em gozo de férias ou de licença no momento da pesquisa. 


\section{Análise de dados}

A análise quantitativa foi realizada com base na estatística descritiva, cujos dados numéricos captados foram armazenados em bancos de dados numéricos do Microsoft Office Excel ${ }^{\circledR}$ e posteriormente interpretados e apresentados em tabelas e quadro.

\section{Resultados}

Foram avaliados 29 profissionais, sendo em sua maioria com tempo de atuação de 1 a 5 anos na Oftalmologia $(65,5 \%)$, no Hospital $(72,4 \%)$ e no setor de Oftalmologia do Hospital (75\%). Desses 29 entrevistados, $68,9 \%$ são atuantes na Urgência/Emergência em Oftalmologia (Tabela 1).

Tabela 1. Perfil dos profissionais do setor de Oftalmologia em um Hospital Universitário em Belém, PA, 2021.

\begin{tabular}{lc}
\hline Variáveis & n (\%) \\
\hline Sexo (F/M) & $20 / 9$ \\
Idade (anos) & $35,7 \pm 8,4$ \\
Tempo de atuação no Hospital (anos) & $21(72,4 \%)$ \\
$1-5$ & $4(13,7 \%)$ \\
$6-10$ & $4(13,7 \%)$ \\
$>10$ & \\
Tempo de atuação em Oftalmologia (anos) & $19(65,5 \%)$ \\
$1-5$ & $7(24,1 \%)$ \\
$6-10$ & $3(10,3 \%)$ \\
$>10$ & \\
Atuação na Urgência/Emergência em Oftalmologia & $20(68,9 \%)$ \\
Sim & $9(31 \%)$ \\
Não & \\
Tempo de atuação no setor de Oftalmologia do Hospital & $15(75 \%)$ \\
$1-5$ & $3(15 \%)$ \\
$6-10$ & $2(10 \%)$ \\
$>10$ &
\end{tabular}

Quanto ao conhecimento dos profissionais sobre Urgências/Emergências do setor de Oftalmologia no HUBFS, 68,9\% acreditam que o HUBFS tem capacidade de atender $\mathrm{U} / \mathrm{E}$ nas 24 horas, 53,5\% disseram que os equipamentos existentes no HUBFS atendem adequadamente um paciente em

Tabela 2. Conhecimento dos profissionais sobre Urgências/Emergências do setor de Oftalmologia em um Hospital Universitário em Belém, PA, 2021.

\begin{tabular}{lcc}
\hline Variáveis & $\begin{array}{c}\text { Sim } \\
\text { n (\%) }\end{array}$ & $\begin{array}{c}\text { Não } \\
\text { n (\%) }\end{array}$ \\
\hline $\begin{array}{l}\text { Capacidade para atender U/E } \\
\text { É nas 24 horas? }\end{array}$ & $20(68,9 \%)$ & $9(31 \%)$ \\
importante que o Hospital atenda U/E nas 24 horas? & $26(96,2 \%)$ & $1(3,7 \%)$ \\
$\begin{array}{l}\text { Os equipamentos existentes no Hospital atendem adequadamente um } \\
\text { paciente em situação de U/E? }\end{array}$ & $15(53,5 \%)$ & $13(46,4 \%)$ \\
$\begin{array}{l}\text { Você tem conhecimento do número de U/E em Oftalmologia atendidos } \\
\text { mensalmente no Hospital? }\end{array}$ & $5(17,2 \%)$ & $24(82,7 \%)$ \\
$\begin{array}{l}\text { Você tem conhecimento do fluxo externo de demanda do atendimento } \\
\text { U/E Oftalmológica? }\end{array}$ & $15(51,7 \%)$ & $14(48,2 \%)$ \\
\hline
\end{tabular}

situação de U/E, 82,7\% desconhecem o número de $\mathrm{U} / \mathrm{E}$ em Oftalmologia atendidos mensalmente no HUBFS e $51,7 \%$ têm conhecimento do fluxo externo de demanda do atendimento U/E Oftalmologia (Tabela 2).

${ }^{\epsilon} \mathrm{U} / \mathrm{E}$ : Urgências/Emergências 
$\mathrm{Na}$ tabela 3, observa-se o conhecimento sobre a capacidade instalada da Urgência e Emergência oftalmológica pelos profissionais do setor do HUBFS. Segundo os entrevistados, 58,6\% acredita que exista um espaço adequado para o atendimento do paciente em situação de U/E no HUBFS, $61,5 \%$ disseram desconhecer protocolo assistencial para $\mathrm{o}$ atendimento de um paciente em situação de U/E no HUBFS. Quanto à priorização do atendimento de U/E em relação aos pacientes ambulatoriais, $82,7 \%$ afirmaram acontecer. E 71,4\% afirmaram que o serviço de Oftalmologia tem acesso imediato ao Centro Cirúrgico em situações que necessitam de intervenção cirúrgica de U/E.

Tabela 3. Conhecimento da capacidade instalada da Urgência e Emergência oftalmológica segundo os profissionais do setor em um Hospital Universitário em Belém, PA, 2021.

\begin{tabular}{lcc}
\hline Variáveis & $\begin{array}{c}\text { Sim } \\
\mathbf{n}(\mathbf{\%})\end{array}$ & $\begin{array}{c}\text { Não } \\
\mathbf{n}(\mathbf{\%})\end{array}$ \\
\hline $\begin{array}{l}\text { Existe um espaço adequado para o atendimento do paciente em situação de U/E* } \\
\text { no Hospital? }\end{array}$ & $17(58,6 \%)$ & $12(41,3 \%)$ \\
$\begin{array}{l}\text { Existe protocolo assistencial para o atendimento de um paciente em situação de } \\
\text { U/E no Hospital? }\end{array}$ & $11(42,3 \%)$ & $16(61,5 \%)$ \\
$\begin{array}{l}\text { O paciente que vem para atendimento de U/E é priorizado em relação aos pacientes } \\
\text { ambulatoriais? }\end{array}$ & $24(82,7 \%)$ & $5(17,2 \%)$ \\
$\begin{array}{l}\text { O serviço de Oftalmologia tem acesso imediato ao Centro Cirúrgico em situações } \\
\text { que necessitam de intervenção cirúrgica de U/E? }\end{array}$ & $20(71,4 \%)$ & $8(28,5 \%)$ \\
\hline${ }^{*}$ U/E: Urgências/Emergências & &
\end{tabular}

Ao questionar sobre as sugestões de mudanças para a melhoria do atendimento da Urgências/Emergências Oftalmológicas pelos profissionais do setor no HUBFS, observaram-se aspectos de Estrutura Pessoal e Física, como pode ser visto no quadro 1.

Quadro 1. Mudanças necessárias segundo os profissionais do setor para a melhoria do atendimento da Urgências/Emergências Oftalmológicas em um Hospital Universitário em Belém, PA, 2021.

\begin{tabular}{|l|l|}
\hline \multicolumn{1}{|c|}{ Estrutura de Pessoal } & \multicolumn{1}{c|}{ Estrutura Física } \\
\hline Redefinir carga horária & $\begin{array}{l}\text { Sala de acolhimento, descanso e refeitório para } \\
\text { profissionais }\end{array}$ \\
\hline Contratar profissionais & Separação de leitos \\
\hline Escala de plantão para preceptores e residentes & Segurança do campus \\
\hline Comprometimento com o serviço & Manutenção contínua dos equipamentos \\
\hline Treinamentos & $\begin{array}{l}\text { Transporte para funcionários e pacientes } \\
\text { Ambulâncias, insumos equipamentos } \\
\text { específicos para U/E }\end{array}$ \\
\hline Melhoria nos protocolos de atendimento & \\
\hline
\end{tabular}

${ }^{*} \mathrm{U} / \mathrm{E}$ : Urgências/Emergências 


\section{Discussão}

As urgências oftalmológicas continuam sendo uma das principais causas de morbidade e cegueiras preveníveis, sendo um dos grandes problemas de saúde pública no mundo, devido à pouca oferta de serviço especializado para essa finalidade. A regulação de acesso é um dos grandes desafios para o sistema de saúde brasileiro, em especial aos serviços oftalmológicos, pois quando estruturada com processos, protocolos e fluxos bem estabelecidos garante a organização do acesso, da oferta e da demanda dos serviços e oferece qualidade e resolutividade na assistência em tempo hábil à população.

Neste trabalho avaliamos a regulação da assistência de urgências e emergências oftalmológicas a partir da ótica dos profissionais da saúde do próprio setor. Observou-se que o tempo de atuação desses profissionais no setor de oftalmologia mantiveram-se nos últimos 5 anos. Sendo assim, podemos supor que a ausência de rotatividade dos profissionais representa um ponto positivo para a qualidade do serviço prestado, pois acredita-se que quanto maior o tempo de permanência do funcionário no setor, maior serão as possibilidades para que este detenha conhecimento dos processos de trabalho. ${ }^{7}$

Entende-se que é importante ter hospitais que atendam as urgências e emergências oftalmológicas com profissionais experientes e capacitados para tal atendimento, pois a estabilidade cria garantias de qualidade nos processos de trabalho, devido esses profissionais já conhecerem os fluxos, normas, protocolos e a estrutura, bem como as patologias atendidas nos serviços. ${ }^{8}$ Por outro lado, a rotatividade é necessária no ambiente hospitalar, pois favorece que os profissionais desenvolvam novos conhecimentos e capacidade técnica para atuar em vários setores, além de promover habilidades, motivação, melhorar relacionamentos interpessoais e auxiliar na redução de conflitos ${ }^{9}$.
Ao analisar o conhecimento dos profissionais sobre o setor de oftalmologia verificou-se que a maioria considera que o hospital tem capacidade para atender U/E oftalmológica nas 24 horas e foi unânime entre os profissionais referir a importância do atendimento nas 24 horas. Contudo, alguns autores têm observado que os pacientes que buscam atendimento apresentam doenças e/ou distúrbios oculares de fácil resolutividade que não necessitariam do pronto-atendimento ${ }^{10}$. O que reforça a necessidade dos profissionais, além de compreenderem a importância do serviço de 24 horas, conhecer as doenças oftalmológicas e aplicar medidas de educação e prevenção para evitar o uso dos serviços especializados de forma inapropriada na unidade ${ }^{11,12}$.

Os profissionais entrevistados consideram que o serviço de oftalmologia apresenta estrutura suficiente para prestação de atendimento adequado em situações de U/E oftalmológica. A visão dos profissionais demonstra que eles reconhecem a importância do serviço. A estrutura para atendimento de urgência e emergência tem forte influência na qualidade do serviço prestado; as instituições de saúde devem garantir estrutura adequada para assistência ao usuário, prevendo os recursos humanos, espaço físico, insumos e recursos tecnológicos ${ }^{13}$.

A maioria dos profissionais desconhecem o número de atendimentos realizados pelo ambulatório. $\mathrm{O}$ conhecimento dessas informações por parte dos profissionais é de extrema relevância para o planejamento das ações que são fundamentais para melhoria do atendimento prestado. A divulgação de dados quantitativos de pacientes em um hospital é muito importante para avalição da qualidade da assistência prestada. Esses indicadores são instrumentos de mensuração que ajudam os profissionais a compreender o tipo de serviço que está 
sendo oferecido ao usuário, o que contribui para melhoria da assistência ${ }^{14}$.

No que se refere ao fluxo de atendimento externo, mais da metade dos trabalhadores têm conhecimento do fluxo dos processos de atendimento. $\mathrm{O}$ fato pode ser atribuído ao tempo de permanência dos profissionais no serviço onde foi verificado a ausência da rotatividade dos profissionais que já atuam nesse setor pelo período de pelo menos 5 anos. Sabe-se que o conhecimento do fluxo de um serviço é de extrema importância para facilitar o acesso do usuário ao atendimento de saúde em especial oftalmológico, uma especialidade pouco ofertada no estado do Pará, no qual a única referência do SUS no Estado é o serviço de oftalmologia do Hospital. O fluxo de atendimento estabelecido em uma instituição é de fundamental importância para a articulação nos diferentes níveis de atenção à saúde, considerando que promove mudança nos processos de trabalho ${ }^{15}$

Segundo os profissionais, observase que o hospital tem espaço adequado para o atendimento de U/E oftalmológica no setor. Fato que é de fundamental importância para a qualidade da assistência. A ambiência hospitalar para um atendimento ao usuário em situação de urgência e emergência ocular necessita ter um espaço específico para esse fim, que facilite o acesso ágil dos pacientes, com equipamentos adequados, equipe multiprofissional capacitada, respeitosa e acolhedora $^{16}$

Ao avaliar o grau de conhecimento dos profissionais em relação ao protocolo assistencial de U/E, a maioria dos profissionais do setor disse desconhecer a existência de um protocolo para atendimento. O desconhecimento sugere que o serviço não possui protocolos implantados. A aplicação diária dos protocolos ocasiona uma assistência segura e padronizada baseada em evidências científicas. Contudo, é essencial para a implantação dos protocolos a educação permanente dos profissionais para 0 incremento da prestação de assistência ${ }^{17}$.
Em relação ao fator prioridade no atendimento, os profissionais consideram em sua maioria que acontecem no serviço, embora tenham relatado a inexistência de protocolos de prioridade descritos; esses profissionais já seguem uma rotina definida de prioridade que é realizada após avaliação médica e que apesar de não estar escrita é de conhecimento de toda equipe. Contudo, é importante a implantação de protocolos e treinamentos com os profissionais do setor com vista à priorização do paciente $\mathrm{e}$ baseada nos princípios da integralidade e equidade. Assim, há um melhor acolhimento do usuário e organização do serviço $^{18}$.

Em relação às sugestões de mudanças para melhoria do atendimento de urgências e emergências oftalmológicas no Hospital, foram descritas mudanças relacionadas à estrutura pessoal e à estrutura física. Contudo, a qualidade de um serviço de urgência oftalmológica é medida não só pelas instalações e desempenho técnico da equipe; a qualidade tem como parâmetros, também, a questão da segurança da equipe no exercício de suas atividades, conforme consta na portaria $288 / 2008^{19}$ do Ministério da Saúde em seu anexo I, que define as exigências mínimas de estrutura física que uma instituição deve ter para atender urgências e emergências em oftalmologia nas 24 horas.

Dispõem ainda entre outros, que os equipamentos devem ter manutenção preventiva e corretiva; ressalta a necessidade de insumos para atendimento ao usuário, além de destacar a obrigação estrutural de refeitórios e salas de repouso para as equipes de acordo com a RDC 50 de 21 de fevereiro de $2002^{20}$.

Os trabalhadores destacam também as mudanças necessárias relacionadas à estrutura física, está deve ter um espaço específico para atendimento das urgências, além de insumos e equipamentos que são fatores que contribuem para assegurar um atendimento rápido $\mathrm{e}$ um tratamento adequado, com diagnóstico precoce das 
urgências e melhoria no prognóstico do paciente $^{16}$.

O conhecimento dos profissionais sobre fluxo, protocolos, capacidade instalada, estrutura física e de pessoal são fatores importantes que interferem de forma direta no atendimento dos pacientes em situação de urgência e emergência oftalmológica, pois contribuem para melhorar o diálogo com os gestores e ajudam a diminuir a alta morbidade e a cegueira prevenível relacionadas aos distúrbios oculares, visto que, de acordo com os resultados encontrados na pesquisa, verificaram-se fragilidades ligadas à qualificação do setor de oftalmologia, seja dos profissionais, seja da estrutura física e da regulação de acesso, que podem ser sanadas através de melhorias na estrutura do Hospital e da organização das redes de atenção oftalmológica, garantindo acesso integral do usuário desde a atenção primária até $\mathrm{o}$ atendimento especializado.

A pesquisa apresentou algumas limitações como o quantitativo de profissionais entrevistados, porém tais limitações não diminuíram a importância do estudo que possibilitou conhecer a realidade da assistência de urgência e emergência prestada por um hospital universitário, sob o ponto de vista da equipe multiprofissional fomentando assim a discussão sobre a habilitação do serviço de U/E oftalmológica.

\section{Conclusão}

Este estudo propôs identificar a regulação do acesso à assistência das $\mathrm{U} / \mathrm{E}$ oftalmológicas sob o ponto de vista dos profissionais do setor e encontrou que a maioria destes, reconhece a importância do serviço para comunidade, desconhece os protocolos, porém destaca a necessidade de melhorias nos processos de trabalho.

Portanto, faz-se necessário uma regulação de acesso fortalecida e com os serviços habilitados conforme as portarias para que se obtenha uma assistência cada vez mais qualificada, em busca da equidade no acesso e eficiência nos serviços.

\section{Referências Bibliográficas}

1. Cortez ACL, Silva CRL, Silva RCL, Dantas EHM. Aspectos gerais sobre a transição demográfica e epidemiológica da população brasileira. Enferm Bras. 2019; 8(5):700709 .

2. Lima NC de, Baptista TW de F, Vargas EP. Ensaio sobre 'cegueiras': itinerário terapêutico e barreiras de acesso em assistência oftalmológica. Interface (Botucatu). 2017;21(62): p.615-27.

3. Viacava F, Oliveira RAD de, Carvalho C de C, Laguardia J, Bellido JG. SUS: oferta, acesso e utilização de serviços de saúde nos últimos 30 anos. Ciênc saúde colet. 2018; 23(6):1751-1762.

4. Cavalcanti RP, Cruz DF da, Padilha WWN. Desafios da regulação assistencial na organização do Sistema Único de Saúde. Rev bras ciênc saúde. 2018;22(2):181-188.

5. Feijo VBElR, Junior LC, Souza RKT de, Dias AO. Análise da demanda atendida em unidade de urgência com classificação de risco. Saúde debate. 2015;39(106):627-636.

6. Barros FPC de, Amaral TCL. Os desafios da regulação em saúde no Brasil. An Inst Hig Med Trop. 2017;16(Supl. 3):S39-S45.

7. Avaristo JAC, Souza ME de. Motivação de pessoas no setor público: uma breve reflexão sobre a literatura. Braz Ap Sci Rev. 2019; 39 (1): 313-332. 
8. Tonelli BQ, Leal AP dos R, Tonelli WFQ, Veloso DCMD, Gonçalves DP, Tonelli SQ. Rotatividade de profissionais da Estratégia Saúde da Família no município de Montes Claros, Minas Gerais, Brasil. Rev Fac Odontol (Univ. Passo Fundo). 2018;23(2):180185.

9. Martins M da S, Matos EM, Salum NC. Rotatividade dos trabalhadores de enfermagem em uma unidade de emergência adulto. Texto Contexto Enferm [Internet]. 2019; 28:e20160069.

10. Pierre Filho P, Gomes PRP, Pierre ETL, Pinheiro Neto FB. Profile of ocular emergencies in a tertiary hospital from Northeast of Brazil. Rev Bras Oftalmol. 2010; 69 (1): 12-7.

11. Campos GM, Brum IV, Brum IV. Perfil epidemiológico dos atendimentos em um serviço público de urgência oftalmológica. Rev Bras Oftalmol. 2019; 78 (5): 297-9.

12. Espinosa PG, Knob HH, Marchetti MP, Stock RA, Rieger A, Bonamigo EL. Arq. Catarin Med. 2020; 49(1):78-90.

13. Cunha VP da, Erdmann AL, Santos JLG dos, Menegon FHA, Nascimento KC do. Atención a pacientes en situación de urgencia: del servicio prehospitalario móvil al servicio hospitalario de emergência. Enferm Actual Costa Rica. 2019;(37), Não paginado.

14. Oliveira RR de. Dos conceitos de regulação às suas possibilidades. Saúde soc. 2014; 23(4):1198-1208.

15. Egry EY, Apostolico MR, Morais TCP. Notificação da violência infantil, fluxos de atenção e processo de trabalho dos profissionais da Atenção Primária em Saúde. Ciênc saúde coletiva. 2018; 23(1):83-92.

16. Santos RWC, Brasileiro ME. Traumas oftalmológicos e a importância do profissional de enfermagem no pré - atendimento. Revista Saúde Integral. 2019;1(3). Não paginado.

17. Sales CB, Bernardes A, Gabriel CS, Brito M de FP, Moura AA de, Zanetti ACB. Protocolos Operacionais Padrão na prática profissional da enfermagem: utilização, fragilidades e potencialidades. Rev bras enferm. 2018;71(1):126-34.

18. Krauzer IM, Dall'Agnoll CM, Gelbckee FL, Lorenzini E, Ferraz L. A construção de protocolos assistenciais no trabalho em enfermagem. Rev Min Enferm. 2018;22: e1087.

19. BRASIL. Ministério da Saúde. Portaria no 288 de 19 de maio de 2008.

20. BRASIL. Ministério da Saúde. Resolução RDC nº 50, de 21 de fevereiro de 2002.

\section{Como citar este artigo:}

Bemerguy CL, Martins ICVS, Guerreiro JF. Regulação de acesso da urgência e emergência oftalmológica em um hospital universitário. Rev. Aten. Saúde. 2021; 19(70): 47-55. 